Draft Version December 1, 2018

Preprint typeset using $\mathrm{LAT}_{\mathrm{E}} \mathrm{X}$ style emulateapj v. 08/22/09

\title{
BENT-DOUBLE RADIO SOURCES AS PROBES OF INTERGALACTIC GAS
}

\author{
E. Freeland, R. F. Cardoso, E. Wilcots \\ Department of Astronomy, University of Wisconsin, Madison, WI 53706 \\ Draft version December 1, 2018
}

\begin{abstract}
As the most common environment in the universe, groups of galaxies are likely to contain a significant fraction of the missing baryons in the form of intergalactic gas. The density of this gas is an important factor in whether ram pressure stripping and strangulation affect the evolution of galaxies in these systems. We present a method for measuring the density of intergalactic gas using bent-double radio sources that is independent of temperature, making it complementary to current absorption line measurements. We use this method to probe intergalactic gas in two different environments: inside a small group of galaxies as well as outside of a larger group at a $2 \mathrm{Mpc}$ radius and measure total gas densities of $4 \pm 1_{-2}^{+6} \times 10^{-3} \mathrm{~cm}^{-3}$ and $9 \pm 3_{-5}^{+10} \times 10^{-4} \mathrm{~cm}^{-3}$ (random and systematic errors) respectively. We use X-ray data to place an upper limit of $2 \times 10^{6} \mathrm{~K}$ on the temperature of the intragroup gas in the small group.

Subject headings: intergalactic medium - galaxies; jets - galaxies: clusters: general
\end{abstract}

\section{INTRODUCTION}

In the local universe, attempts to measure the baryon content in the form of stars, hot X-ray emitting gas, and cold gas account for only one third of the baryon density seen at high redshift (Fukugita \& Peebles 2004). Simulations predict that the remaining "missing baryons" exist in a warm-hot intergalactic medium (WHIM) that was shock heated during large-scale structure formation to a temperature range of $10^{5}<T<10^{7} \mathrm{~K}$ (Cen \& Ostriker 2006; Davé et al. 2001). Observational confirmation of the WHIM comes primarily from UV absorption lines in the spectra of low redshift quasars (Tripp et al. 2000). However, UV absorption line observations are only able to probe a limited temperature range of WHIM gas and there is continued debate as to whether the absorption originates in the extended gaseous halos of individual galaxies, the intergalactic medium (IGM), or from largescale filaments (Savage et al. 2003).

Galaxy groups, the most common environment in the local universe, likely contain a significant fraction of the total baryonic mass within their intragroup medium (Fukugita et al. 1998). Until now the only probes of this gas have been UV absorption line and X-ray observations. In the few groups that have a hot intragroup medium X-ray observations can determine the emission measure, but without some knowledge of the geometry the density of this gas cannot be disentangled. The X-ray surface brightness typically falls below detectable levels at radii much smaller than the virial radius, while the implied gas mass is still increasing at least linearly with radius (Mulchaev 2000). Groups with a bright quasar behind them have been observed in an attempt to detect absorption from the intragroup medium. In cases where intervening gas is detected, assumptions about the extent of the absorbing system, the metallicity, ionization fraction, and the intensity of the ionizing radiation field lead to estimates of the intragroup medium density on the order of $n \sim 10^{-4}-10^{-5} \mathrm{~cm}^{-3}$ (Pisano et al. 2004;
Tripp et al. 1998).

Additionally, galaxy groups, according to the hierarchical scenario of the formation of large-scale structure, are the building blocks of rich clusters of galaxies. Groups are important sites in which to investigate the physical mechanisms responsible for the observed morphology and SFR-density relations (Goto et al.|2003; Gómez et al. 2003). However, the effectiveness of mechanisms like ram pressure stripping and strangulation on galaxy evolution in the group environment are not well understood due to the uncertainty in the density of the intragroup medium. Some observational evidence does exist for the stripping of hot gas from galaxies by the intragroup medium, mainly in features like X-ray shocks and tails (Sivakoff et al. 2004; Rasmussen et al.|2006), as well as HI observations that show compression features opposite extensions in cold disk gas (Kantharia et al. 2005; : McConnachie et al. 2007).

Another way to measure the density of the IGM is through its effect on the jets of radio galaxies. As a double lobed radio source travels through intergalactic gas ram pressure causes its jets to be swept back. Prior to Burns et al. (1987) it was believed that the combination of the dense intracluster medium and large galaxy velocities needed to form these bent-double radio sources could only be found in rich clusters. While many bent-doubles do reside in dense clusters, a surprising number are found in lower mass environments like groups of galaxies (Ekers et al. 1978; Venkatesan et al. 1994; Doe et al. 1995; Blanton et al.|2001).

We present measurements of the density of intergalactic gas from radio, optical, and X-ray observations of two bent-double radio sources and their surrounding environments. Both sources were identified by Blanton et al. (2001) as existing in environments with richness less than Abell class 0 . We adopt $H_{0}=73 \mathrm{~km} \mathrm{~s}^{-1} \mathrm{Mpc}^{-1}$.

Electronic address: freeland@astro.wisc.edu, cardoso@astro.wisc.edu, ewilcots@astro.wisc.edu 1 . Radio Observations 
We observed the FRI radio galaxies FIRST J124942.2+ 303838 and SDSS J085331.86 + 232400.0 (hereafter S1 and S2 respectively) in March of 2006 with the Giant Metrewave Radio Telescope (GMRT). The GMRT consists of thirty 45 meter diameter antennas with half arranged in a compact $1 \mathrm{~km}$ diameter, randomly distributed, central square and the remaining in three extended arms with fixed baselines ranging from 0.1 to $25 \mathrm{~km}$ in length. The observations were dual frequency $610 / 235 \mathrm{MHz}$ using both the upper and lower sidebands. In this paper we will discuss only the single polarization $610 \mathrm{MHz}$ data which have an effective bandwidth of $12 \mathrm{MHz}$ in each sideband. We observed a flux calibrator (3C286 or 3C48) at the beginning and end of each observation and every 40 minutes we observed a phase calibrator for 6 minutes. We have a total of 5.5 hours integration on each source. The data were calibrated in AIPS using standard tasks. Imaging and self-calibration were done with multiple facets across the $0.7^{\circ}$ (half-power beam width) field of view.

\subsection{Optical Observations}

Multi-object spectroscopy was performed in May 2007 and April 2008 using HYDRA on the WIYN 3.5m telescope on Kitt Peak for the source S1. Fibers from the blue cables were placed on galaxies chosen from Sloan Digital Sky Survey (SDSS) photometric redshifts to have a redshift similar to the radio source, and additional fibers were placed on blank sky positions. The 600@10 Zepf grating was used in first order to give spectra with a dispersion of $1.4 \AA$ per pixel covering a wavelength range from 4600 to $7200 \AA$. CuAr lamp calibration spectra were used for the wavelength calibration and an averaged sky spectrum was subtracted from each object spectrum. Spectra were obtained for $\sim 60$ galaxies overall. The data were reduced in IRAF using the dohydra package. Cross correlation of object spectra with galaxy template spectra was performed using the RVSAO (Kurtz \& Mink 1998) package in IRAF.

\subsection{X-ray Observations}

We obtained archival Chandra observations of both sources taken originally in January and February 2005 (PI Blanton). Exposure times were 35.17 and $47.19 \mathrm{ksec}$ for S1 and S2, respectively, and both sources were centered on the back illuminated ACIS-S3 chip.

The event files were filtered to include events with energies between $0.3 \mathrm{keV}$ and $7.0 \mathrm{keV}$. Point sources were identified using task wavdetect and removed. Images were made by smoothing the data in two dimensions with a gaussian extending to $5 \sigma$ and with a full-width at half maximum of 6 pixels on both axes. Images were also made using an adaptive smoothing with a minimal significance signal to noise ratio of 3 . Spectra were extracted from a region $300 \mathrm{kpc}$ in radius around the radio source as well as a background region. These sources are distant enough that they fit on the back illuminated ACIS-S3 chip with area left for a generous background region.

\section{METHOD}

For a relativistic, hydrodynamic jet, the timeindependent Euler equation describes the balance of in- ternal and external pressure gradients (Begelman et al. 1979; Jones \& Owen 1979; Burns \& Owen 1980),

$$
\frac{\rho_{\mathrm{IGM}} v_{g a l}^{2}}{h}=\frac{\mathrm{w} \Gamma^{2} \beta^{2}}{R}
$$

where $\rho_{\mathrm{IGM}} v_{\text {gal }}^{2}$ is the external ram pressure felt by the radio galaxy as it travels through the IGM, $\mathrm{w} \Gamma^{2} \beta^{2}$ is the relativistic enthalpy density inside the jet, $h$ is the width of the jet, and $R$ is the radius of curvature of the jet. In our data the jet widths are not resolved and are set by the beam size of the individual observations. The radius of curvature, $R$, is found by fitting a circle by eye along the jets through the core. This radius is illustrated in the left panels of Figure 1. The velocity of the radio galaxy, $v_{\text {gal }}$, is estimated using the velocity dispersion of the group as $\sqrt{3} \sigma_{v}$. The enthalpy density is written as $\mathrm{w}=e+p$, where $e$ is the energy density and $p$ is the internal pressure. For the jets whose particle population is ultrarelativistic, $e=3 p+\rho_{\text {jet }} c^{2}$ and thus $\mathrm{w}=4 P_{\min }+\rho_{\text {jet }} c^{2}$ assuming that the internal pressure in the jets is dominated by the minimum synchrotron pressure, $P_{\min }$. While $\rho_{j e t} c^{2}$ is not well known, it is anticipated to be much less than $4 P_{\min }$ and we do not include it in our calculations (Bridle \& Perlev 1984). We adopt $\beta=v / c=0.54 \pm 0.18$ as the distribution of observed jet speeds in FRI radio galaxies with straight jets (Arshakian \& Longair 2004) which is consistent with speeds in wide-angle tailed radio galaxies (Jetha et al. 2006). The relativistic factor $\Gamma$ is the usual $\left(1-\beta^{2}\right)^{-1 / 2}$.

The internal pressure is calculated using the minimum synchrotron pressure as outlined in O'Dea \& Owen (1987).

$P_{\min }=(2 \pi)^{-\frac{3}{7}}\left(\frac{7}{12}\right)\left[c_{12} L_{\mathrm{rad}}(1+k)(\phi V)^{-1}\right]^{\frac{4}{7}} \mathrm{ergs} \mathrm{cm}^{-3}$

where $c_{12}$ is a constant that depends on the spectral index and frequency cutoffs (Pacholczyk 1970), $k$ is the ratio of relativistic proton to relativistic electron energy, $\phi$ is the volume filling factor, $V$ the source volume, and $L_{\mathrm{rad}}$ the radio luminosity of the jet. We measure this internal pressure at the position immediately before the jet bends, always excluding the core. Standard equipartition of energy between relativistic particles and magnetic fields is assumed. We follow the general assumptions that $k=1$, $\phi=1$, and the jets are cylinders uniformly filled with magnetic fields and relativistic particles. We use VLA FIRST survey (Becker et al. 1995) data at $1420 \mathrm{MHz}$ and our GMRT $610 \mathrm{MHz}$ data to determine the spectral index of the synchrotron emission. Our values for the minimum synchrotron pressure in the jets are in good agreement with other measurements for similar radio sources (Venkatesan et al. 1994; Worrall \& Birkinshaw 2000).

\section{GROUP REDSHIFTS AND VELOCITY DISPERSIONS}

In order to estimate the velocity of the radio galaxy through the IGM we use the optical data to examine the environments of these sources. Small group memberships and instrumentation limit our ability to obtain large radial velocity samples. Additionally, structures associated with these radio sources may not have achieved the 
dynamical equilibrium that would result in a Gaussian distribution of radial velocities among members. With these considerations in mind we use the robust statistical biweight estimators for location and scale (Beers et al. 1990) to determine the group redshift and velocity dispersion for each sample. These estimators do not assume a Gaussian parent population and are not strongly influenced by outliers which is especially important for small samples.

We combined redshift information from our WIYN spectroscopy (only for S1), as well as spectroscopic and photometric redshifts from the Sloan Digital Sky Survey DR6 (Adelman-McCarthy et al. 2008). We use only photometric redshifts whose errors are less than $5 \%$ of their value for $\mathrm{S} 1$ and $10 \%$ for $\mathrm{S} 2$.

We calculate a peculiar velocity for a galaxy with redshift $z$ in the rest frame of the group using

$$
v_{\text {pec }}=c\left(z-z_{\text {group }}\right) /\left(1+z_{\text {group }}\right) .
$$

The velocity dispersion, $\sigma_{\text {group }}$, will be taken as the dispersion of the $v_{p e c}$ values. We start by examining the redshift histogram for galaxies within a projected $6 \mathrm{Mpc}$ distance from the radio source and the sky locations of galaxies within $2000 \mathrm{~km} \mathrm{~s}^{-1}$. With so few redshifts available the final samples are chosen by eye based on the observed spatial clustering seen in Figure 1 which shows the spatial and redshift distributions of galaxies near the two radio sources. In the case of $\mathrm{S} 2$, the radio source appears offset spatially from the group. 10 of the galaxies circled in black have photometric redshifts and 3 (including the radio source) are SDSS spectroscopic redshifts. We estimate the speed of this source through the IGM using its velocity relative to the mean redshift of the system it is approaching $(\bar{z}=0.3081)$ and find a speed of $570 \mathrm{~km} \mathrm{~s}^{-1}$. The velocity of this source is uncertain although not unreasonable and we use a rough error of $10 \%$ of its value in future calculations. S1 is associated with four other nearby galaxies, three have redshifts measured from our WIYN data and the fourth is a photometric redshift from SDSS. These five galaxies have a velocity dispersion of $250_{-110}^{+20} \mathrm{~km} \mathrm{~s}^{-1}$. The $68 \%$ error bars on the velocity dispersion are determined by generating bootstrap samples using the final set of $v_{p e c}$ values.

\section{IGM TEMPERATURE}

We use the X-ray data to examine the temperature of the IGM in these systems. XSPEC (Arnaud 2004) is used to fit the spectra extracted from a region 300 $\mathrm{kpc}$ in radius surrounding the radio source with point sources removed. We use Raymond models for thermal X-ray emission from a hot, optically thin gas, fixing the abundance at $\mathrm{Z}=0.1$ and the normalization according to the measured number density shown in Table 1. assuming it is constant throughout the volume. All models included a WABS component of Galactic absorption with values of $1.25 \times 10^{20} \mathrm{~cm}^{-2}$ and $3.23 \times 10^{20} \mathrm{~cm}^{-2}$ for S1 and S2 respectively, based on the measurements of Dickey \& Lockman (1990).

In the case of S1 the number of counts in the region around the radio source after background subtraction is only $80 \pm 35$. The spectrum from this region was binned to give each measurement a $3 \sigma$ significance level above the background. A Raymond model was then compared with the data to determine the temperature the gas would need to produce counts at a $3 \sigma$ level above the current observations. Gas with a density of $4 \times 10^{-3} \mathrm{~cm}^{-3}$ and a temperature higher than $0.2 \mathrm{keV}\left(2 \times 10^{6} \mathrm{~K}\right)$ should be observable with the current data. Thus, we put an upper limit on the temperature of the IGM in this group at $2 \times 10^{6} \mathrm{~K}$.

After background subtraction we detect $94 \pm 26$ counts from the source region for S2. We do not expect the intergalactic gas around this source to be hot since it is 2 Mpc from the center of a system of galaxies. The adaptively smoothed image shows the X-ray counts concentrated near the radio source. We interpret these X-rays as originating from Inverse-Compton scattering off the relativistic electrons in the radio source. The data are not adequate to distinguish between thermal and nonthermal models for the emission. Additionally, with the current data the density of this gas is too low to place an upper limit on the temperature were we to assume that these X-rays originated from a hot IGM.

\section{DISCUSSION}

We present two measurements of the density of intergalactic gas using radio sources whose jets are bent back by ram pressure. S1 is probing gas with a density of $4 \pm 1_{-2}^{+6} \times 10^{-27} \mathrm{~g} \mathrm{~cm}^{-3}\left(4 \pm 1_{-2}^{+6} \times 10^{-3} \mathrm{~cm}^{-3}\right.$ assuming a mean molecular weight of 0.6 and where the first set of errors are random and the second systematic) that is near the center of a small group of galaxies. In the case of $\mathrm{S} 2$ this source is probing gas that is a projected distance of $2 \mathrm{Mpc}$ from the center of a system of galaxies with a density of $9 \pm 3_{-5}^{+10} \times 10^{-28} \mathrm{~g} \mathrm{~cm}^{-3}\left(9 \pm 3_{-5}^{+10} \times 10^{-4} \mathrm{~cm}^{-3}\right)$. We have not accounted for thermal pressure in the jets, as well as our inability to resolve their true width. Additional thermal pressure or smaller jets will necessitate a larger IGM density to produce the observed jet geometry. If we have overestimated the radio galaxy speed or projection effects have led us to a smaller radius of curvature then the necessary density will decrease.

The systematic errors reflect the range of IGM densities possible given the range of jet speeds discussed in Section 3. These speeds are measured by assuming that flux asymmetries between the jet and counter-jet are the result of relativistic beaming effects. This beaming depends on the angle of the jets from our line of sight as well as the jet speed. By looking at large samples of objects and assuming a uniform distribution of angles, a range of jet speeds can be determined. We lack the inclination information necessary to determine jet speeds for individual sources. Arshakian \& Longair (2004) point out that their distribution of jet speeds is asymmetric with the peak toward lower speeds and that selection effects may be pushing the mean toward higher speeds. Slower jet speeds would cause our IGM densities to tip towards the lower end of the range in values.

The inclination of the source also affects the measured radius of curvature. We use a value which is uncorrected for inclination to calculate the IGM densities. S1 appears relatively unprojected whereas $\mathrm{S} 2$ is less easily characterized. If we assume that a Hubble flow distance is accurate for $\mathrm{S} 2$, and that it is traveling along the vector connecting its current position with the cross indicating the center 
of the nearby system of galaxies, then its inclination from the plane of the sky is $\sim 75^{\circ}$. Deprojecting according to this angle leads to a radius of curvature four times smaller and a corresponding IGM density four times larger.

We can compare the measured IGM densities to those seen in clusters and X-ray bright groups of galaxies where the radial density profile of the hot gas in the IGM is traced using X-ray data. Gas densities at the center of clusters are on the order of $10^{-25} \mathrm{~g} \mathrm{~cm}^{-3}\left(0.1 \mathrm{~cm}^{-3}\right)$ to $10^{-28} \mathrm{~g} \mathrm{~cm}^{-3}\left(10^{-4} \mathrm{~cm}^{-3}\right)$ at a radius of $1 \mathrm{Mpc}$ (Vikhlinin et al. 2006). The IGM density we measure near S1 is comparable to the density seen in X-ray observations at a similar radius in the NGC 5044, NGC 533, and ESO 5520200 groups from Gastaldello et al. (2007). Those groups have higher velocity dispersions and may be dynamically older than the group containing S1.

Numerical simulations of the evolution of the IGM predict WHIM gas in the local universe with a density distribution that is broadly peaked at overdensities of $10-20$ and densities in filaments of $10-100$ relative to the critical density $\left(1.0 \times 10^{-29} \mathrm{~g} \mathrm{~cm}^{-3}\right.$ for $H_{0}=$ $73 \mathrm{~km} \mathrm{~s}^{-1} \mathrm{Mpc}^{-1}$ ) (Cen \& Ostriker 2006; Dolag et al. 2006). The gas that S2 is tracing has an overdensity of $\sim 90$. Considering that this source is $2 \mathrm{Mpc}$ from the center of a large group of galaxies it could be tracing gas in a filament or gas that is only tenuously associated with the nearby group.

Radio galaxies with bent jets in groups of galaxies provide evidence that intragroup gas exists in these systems even when the IGM is not X-ray bright. The densities derived in this paper indicate that groups of galaxies and their surroundings are likely to contain significant reservoirs of baryons. Further work is underway with a larger number of sources and will attempt to quantify the contribution of the intragroup medium to the baryon density in the local universe.

EF would like to acknowledge the support of a Wisconsin Space Grant Consortium Graduate Fellowship. EW was supported by NSF grant AST-0506628. This research has made use of the NASA/IPAC extragalactic database (NED) which is operated by the Jet Propulsion Laboratory, Caltech, under contract with the National Aeronautics and Space Administration. We thank the staff of the GMRT who have made these observations possible. GMRT is run by the National Centre for Radio Astrophysics of the Tata Institute of Fundamental Research.

\section{REFERENCES}

Adelman-McCarthy et al. 2008, ApJS, 175, 297

Arnaud, K. 2004, in Bulletin of the American Astronomical Society, Vol. 36, Bulletin of the American Astronomical Society, 934-+

Arshakian, T. G. \& Longair, M. S. 2004, MNRAS, 351, 727

Becker, R. H., White, R. L., \& Helfand, D. J. 1995, ApJ, 450, 559

Beers, T. C., Flynn, K., \& Gebhardt, K. 1990, AJ, 100, 32

Begelman, M. C., Rees, M. J., \& Blandford, R. D. 1979, Nature, 279, 770

Blanton, E. L., Gregg, M. D., Helfand, D. J., Becker, R. H., \& Leighly, K. M. 2001, AJ, 121, 2915

Bridle, A. H. \& Perley, R. A. 1984, ARA\&A, 22, 319

Burns, J. O., Hanisch, R. J., White, R. A., Nelson, E. R., Morrisette, K. A., \& Moody, J. W. 1987, AJ, 94, 587

Burns, J. O. \& Owen, F. N. 1980, AJ, 85, 204

Cen, R. \& Ostriker, J. P. 2006, ApJ, 650, 560

Davé et al. 2001, ApJ, 552, 473

Dickey, J. M. \& Lockman, F. J. 1990, ARA\&A, 28, 215

Doe, S. M., Ledlow, M. J., Burns, J. O., \& White, R. A. 1995 AJ, 110,46

Dolag, K., Meneghetti, M., Moscardini, L., Rasia, E., \& Bonaldi, A. 2006, MNRAS, 370, 656

Ekers, R. D., Fanti, R., Lari, C., \& Ulrich, M.-H. 1978, A\&A, 69, 253

Fukugita, M., Hogan, C. J., \& Peebles, P. J. E. 1998, ApJ, 503, 518

Fukugita, M. \& Peebles, P. J. E. 2004, ApJ, 616, 643

Gastaldello, F., Buote, D. A., Humphrey, P. J., Zappacosta, L., Bullock, J. S., Brighenti, F., \& Mathews, W. G. 2007, ApJ, 669,158

Gómez et al. 2003, ApJ, 584, 210

Goto, T., Yamauchi, C., Fujita, Y., Okamura, S., Sekiguchi, M. Smail, I., Bernardi, M., \& Gomez, P. L. 2003, MNRAS, 346, 601
Jetha, N. N., Hardcastle, M. J., \& Sakelliou, I. 2006, MNRAS, 368,609

Jones, T. W. \& Owen, F. N. 1979, ApJ, 234, 818

Kantharia, N. G., Ananthakrishnan, S., Nityananda, R., \& Hota, A. 2005, A\&A, 435, 483

Kurtz, M. J. \& Mink, D. J. 1998, PASP, 110, 934

McConnachie, A. W., Venn, K. A., Irwin, M. J., Young, L. M., \& Geehan, J. J. 2007, ApJ, 671, L33

Mulchaey, J. S. 2000, ARA\&A, 38, 289

O'Dea, C. P. \& Owen, F. N. 1987, ApJ, 316, 95

Pacholczyk, A. G. 1970, Radio astrophysics. Nonthermal processes in galactic and extragalactic sources (Series of Books in Astronomy and Astrophysics, San Francisco: Freeman, 1970)

Pisano, D. J., Wakker, B. P., Wilcots, E. M., \& Fabian, D. 2004, AJ, 127, 199

Rasmussen, J., Ponman, T. J., \& Mulchaey, J. S. 2006, MNRAS, 370,453

Savage, B. D., Sembach, K. R., Wakker, B. P., Richter, P., Meade, M., Jenkins, E. B., Shull, J. M., Moos, H. W., \& Sonneborn, G. 2003, ApJS, 146, 125

Sivakoff, G. R., Sarazin, C. L., \& Carlin, J. L. 2004, ApJ, 617, 262

Tripp, T. M., Lu, L., \& Savage, B. D. 1998, in ASP Conf. Ser. 143: The Scientific Impact of the Goddard High Resolution Spectrograph, ed. J. C. Brandt, T. B. Ake, \& C. C. Petersen, $261-+$

Tripp, T. M., Savage, B. D., \& Jenkins, E. B. 2000, ApJ, 534, L1

Venkatesan, T. C. A., Batuski, D. J., Hanisch, R. J., \& Burns, J. O. 1994, ApJ, 436, 67

Vikhlinin, A., Kravtsov, A., Forman, W., Jones, C., Markevitch, M., Murray, S. S., \& Van Speybroeck, L. 2006, ApJ, 640, 691 Worrall, D. M. \& Birkinshaw, M. 2000, ApJ, 530, 719 
TABLE 1

Source Information

\begin{tabular}{lccccccccc}
\hline \hline Source & $\begin{array}{c}\alpha \\
(\mathrm{J} 2000)\end{array}$ & $\begin{array}{c}\delta \\
(\mathrm{J} 2000)\end{array}$ & $\mathrm{z}$ & $\begin{array}{c}L_{1440} \\
\mathrm{~W} \mathrm{~Hz}^{-1}\end{array}$ & $\begin{array}{c}h \\
(\operatorname{arcsec})\end{array}$ & $\begin{array}{c}R \\
(\operatorname{arcsec})\end{array}$ & $\begin{array}{c}v_{\text {gal }} \\
\left(\mathrm{km} \mathrm{s}^{-1}\right)\end{array}$ & $\begin{array}{c}P_{\text {min }, j e t} \\
\left(\mathrm{dynes} \mathrm{cm}^{-2}\right)\end{array}$ & $\begin{array}{c}n_{\text {IGM }} \\
\left(\mathrm{cm}^{-3}\right)\end{array}$ \\
\hline S1 & 124942.2 & 303838 & 0.194 & $1.61 \times 10^{25}$ & 4.1 & $13 \pm 1$ & $250_{-100}^{+20}$ & $2 \times 10^{-11}$ & $\begin{array}{c}4 \pm 1_{-2}^{+6} \times 10^{-3} \\
\text { S2 }\end{array}$ \\
\hline
\end{tabular}

Note. $-L_{1440}$ is from Blanton et al. (2001). 

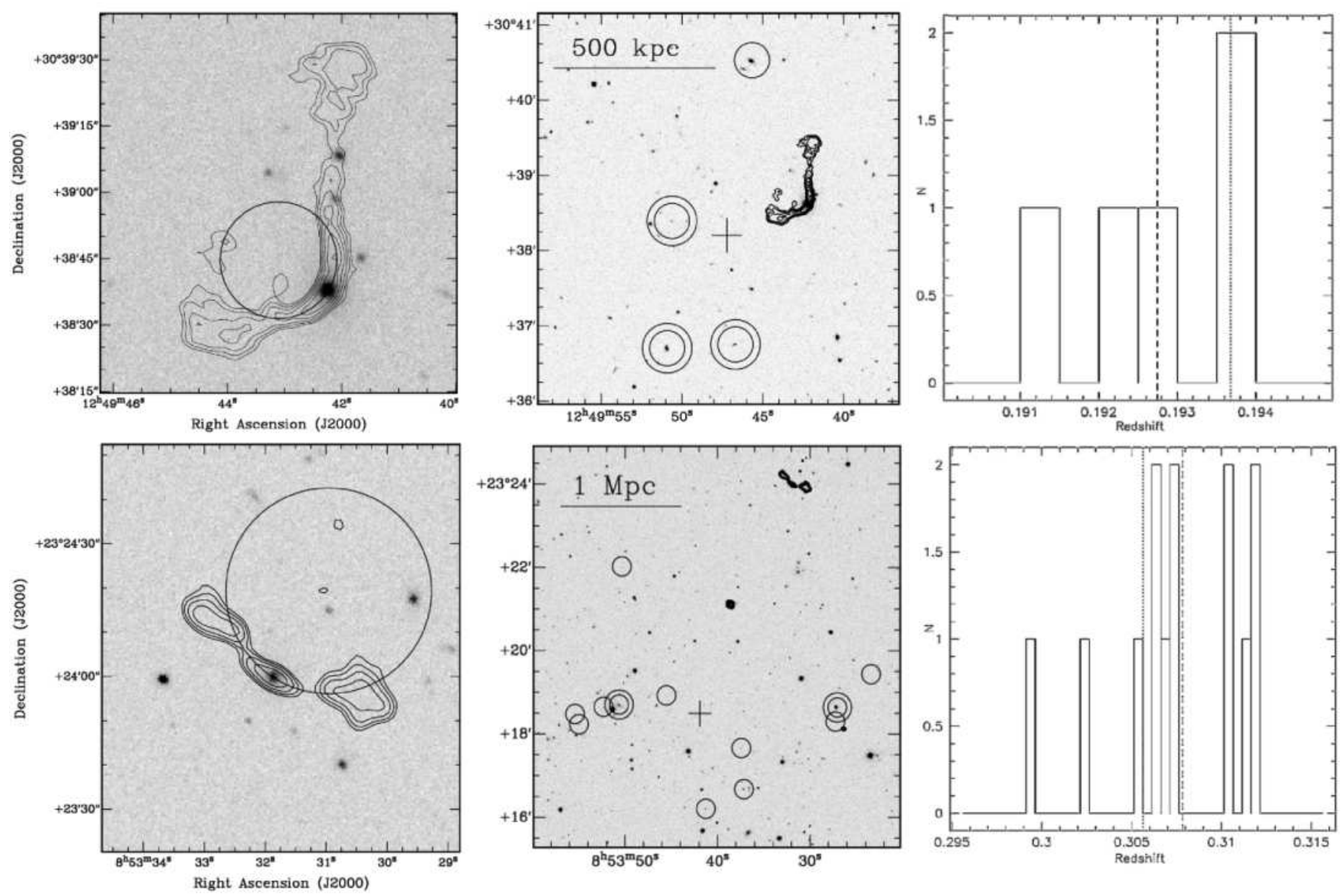

Fig. 1. - $610 \mathrm{MHz}$ GMRT radio contours overlaid on SDSS r band optical images, S1 (top) and S2 (bottom). Lowest contour levels are $1 \mathrm{mJy}_{\text {beam }}^{-1}(\mathrm{~S} 1)$ and $1.3 \mathrm{mJy}_{\text {beam }}{ }^{-1}(\mathrm{~S} 2)$ and increase by $\sqrt{2}$. Beam sizes are $4.3^{\prime \prime} \times 3.3^{\prime \prime}$ for $\mathrm{S} 1$ and $5.8^{\prime \prime} \times 4.9^{\prime \prime}$ for $\mathrm{S} 2$. In the panels on the left the radius of curvature is illustrated. In the center panels, crosses indicate the averaged group center and galaxies with spectroscopic redshifts are circled twice while photometric redshifts are circled once. To the right, the redshift histograms have a dashed line indicating the group redshift and a dotted line at the redshift of the bent-double radio source. 